\title{
Essais d'aptitude au traitement des sédiments de dragage marins en vue de leur valorisation en techniques routières
}

\author{
MAHERZI Walid et BENZERZOUR Mahfoud \\ Ecole des Mines de Douai, Département Génie Civil \& Environnemental 764, Bd Lahure, 59508 Douai, France \\ BEN ABDELGHANI Farouk \\ ISTEUB, rue de l'artisanat, Cherguia II, 2035 Tunis, Tunisie
}

Auteur/s à qui la correspondance devrait être adressée : walid.maherzi@mines-douai.fr

\section{RESUME}

Les résultats présentés dans cet article s'inscrivent dans le cadre du projet de recherche Européen SETARMS dont l'un des objectifs consiste à trouver des solutions innovantes et durables pour la gestion des sédiments marins dragués en Manche (FranceAngleterre) pour une application en techniques routières, plus particulièrement en couche de forme. Après une lère étape de caractérisation géotechnique des sédiments de dragage selon le GTR, les recommandations du GTS ont été utilisées pour le traitement des sédiments aux liants hydrauliques. Ainsi une formulation à $3 \%$ de chaux et $6 \%$ de ciment est appliquée à huit sédiments. Des essais d'aptitude au traitement comprenant des mesures de gonflements volumiques (Gv) et de résistances à la traction indirecte (Rit) ont été réalisés sur les huit sédiments traités en adoptant les normes standards en vigueur en France. Les résultats obtenus montrent que malgré une même origine des sédiments et des classes GTR assez proches (A I etA2), les sédiments traités répondent différemment aux essais d'aptitude. Ces premiers résultats, utiles à l'avancement du projet, permettent une meilleure compréhension de la problématique de traitement des sédiments aux liants hydrauliques et leur valorisation en techniques routières.

MOTS-CLÉS : Sédiments de dragage, essais d'aptitude, liants hydrauliques, traitement, techniques routières.

\begin{abstract}
In this paper, results for an European project called SETARMS which aims to find innovative and sustainable solutions for marine dredged sediments in order to reuse them in road construction. After a first stage of a geotechnical characterization of sediments according to the GTR, the GTS recommendations were adopted to treat sediments with hydraulic binders. Hence, a formulation composed of $3 \%$ of quicklime and $6 \%$ of cement was applied for height different sediments. Specific ability tests were conducted in order to measure volumetric swelling and indirect tensile strength. Obtained results show that even if raw sediments belong to the same GTR class (Al or A2), the treated samples respond differently to the adopted treatment formulation. Results of mechanical performance with the cure age show that most sediments don't reach the needed criteria for reuse as sub-grade layer.
\end{abstract}

KEYWORDS : Dredged sediments, ability test, treatment, hydraulic binders, road construction. 


\title{
Essais d'aptitude au traitement des sédiments de dragage marins en vue de leur valorisation en techniques routières
}

\author{
MAHERZI Walid, BENZERZOUR Mahfoud et BEN ABDELGHANI Farouk
}

\section{INTRODUCTION}

Les activités de dragage visant à étendre et à maintenir la navigabilité des zones portuaires de la Manche revêtent une importance économique capitale. La gestion des matériaux de dragage générés par cette activité est confrontée à des difficultés juridiques, financières, environnementales, techniques et scientifiques. C'est dans ce contexte que le projet Européen SETARMS (Sustainable Environmental Treatment and Reuse of Marine Sediment) a été proposé et sélectionné dans le cadre européen de coopération transfrontalière INTERREG IVA France (Manche)-Angleterre. Dans ce projet, la technique de solidification / stabilisation à base de liants hydrauliques est utilisée afin de valoriser et réutiliser les sédiments de dragage traités. Ce projet, de par sa structure partenariale et ses objectifs, permettra de trouver des solutions économiques et environnementales au dragage, basées sur des travaux scientifiques et techniques. Privilégiant les traitements aux liants hydrauliques et la valorisation en techniques routières (souscouches ou remblais), le projet opérationnel SEDIMARD 83 s'est focalisé sur des traitements de sédiments par inertage. Les résultats de l'étude de valorisation des sédiments ainsi traités (SANNIER et al., 2009 ; LEVACHER et al., 201 I) montrent que les performances mécaniques (seuil de IMPa exigé pour la résistance à la compression simple à 28 jours) associées à une approche environnementale et économique sont atteintes avec plusieurs combinaisons de prétraitement et de traitement. Dans le cadre du projet européen SETARMS, la méthodologie de valorisation des sédiments comme matériau routier (plus particulièrement en couche de forme) repose sur les recommandations des guides techniques LCPC-SETRA (2000) de terrassements routiers et de traitement des sols à la chaux et aux liants hydrauliques.

Les résultats analysés ici concernent les essais d'aptitude au traitement des sédiments. Les réponses des sédiments étudiés en terme d'aptitude au traitement et de comportement mécanique seront analysées afin de dégager des tendances et des corrélations entre les propriétés géotechniques d'un côté et l'aptitude au traitement aux liants hydrauliques et donc à la valorisation de l'autre côté. Ces essais comprennent les mesures de gonflements volumiques (Gv), la détermination des résistances à la traction indirecte (Rit) ainsi que le suivi des performances mécaniques par mesure de la résistance à la compression simple de huit sédiments traités aux liants hydrauliques. Les résultats obtenus montrent que les sédiments ne sont pas tous aptes au traitement adopté et n'atteignent pas les performances mécaniques exigées par le GTS.

\section{Matériaux et méthodes}

Les sédiments de dragage étudiés ont été prélevés dans des ports français et anglais des deux côtés de la Manche, entre ports de plaisance et ports de commerce. Les sites de prélèvement sont localisés sur la carte de la Figure I. Au total, I3 sédiments ont été prélevés dans le cadre du projet européen SETARMS, huit sédiments du côté français et cinq sédiments du côté britannique.

Une première étape de caractérisation géotechnique menée sur des sédiments bruts a permis de classer ces huit sédiments selon le GTR en sols fins Al ou A2, faiblement à fortement organiques FII ou FI2 (Ben Abdelghani et al., 20l4). Le Tableau I récapitule la classification obtenue des cinq différents sédiments qui ont été étudiés.

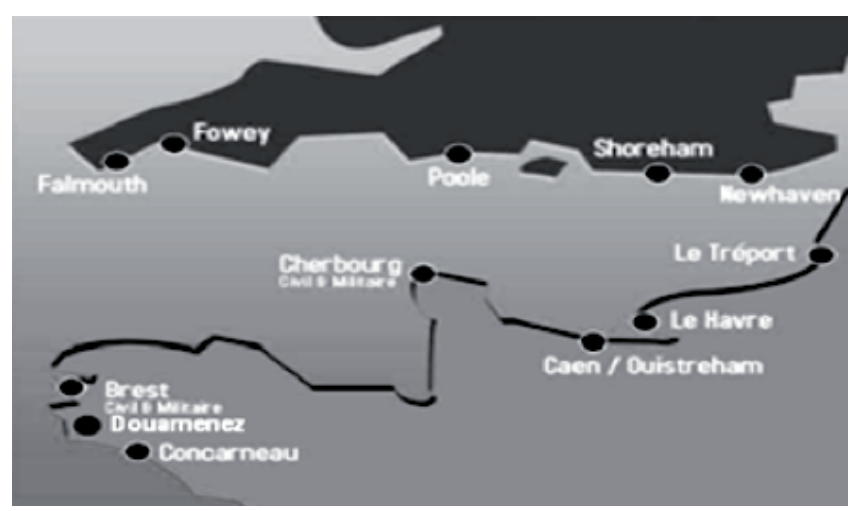

Figure I. Localisation géographique des différents ports d'étude (projet européen SETARMS).

À la lumière de la classification GTR des différents sédiments étudiés, le traitement à adopter est un traitement mixte à base de chaux et de liant hydraulique. La chaux utilisée est une chaux vive (de marque Proviacal@DS) qui répond aux exigences de la norme NF P 98-10 I. Elle est composée de plus de $80 \%$ de chaux ( $\mathrm{CaO}$ ), 5 \% d'oxyde de magnésium (MgO) 
Tableau I. classification gtr des differents sediments etudies.

\begin{tabular}{|c|c|c|c|c|c|c|c|c|}
\hline $\begin{array}{l}\text { Paramètre de } \\
\text { classification }\end{array}$ & $\mathrm{A} 00 \mathrm{I}$ & B00I & $\mathrm{COOI}$ & D00I & E00I & F00I & MOOI & G00 I \\
\hline $\begin{array}{l}\text { Classification GTR } \\
\text { (LCPC-SETRA, 1992) }\end{array}$ & $A_{2} S F_{11}$ & $A_{1} h F_{11}$ & $\mathrm{~A}, \mathrm{~m} F \mid 2$ & $A_{1} m F_{11}$ & $A_{1} h F_{12}$ & $\mathrm{~A}_{2} \mathrm{~m} \mathrm{~F}_{12}$ & $\mathrm{~A}_{2}$ thF $F_{12}$ & $A_{1} h F_{11}$ \\
\hline
\end{tabular}

et de 0 à $5 \%$ de constituants secondaires. La chaux vive a été utilisée dans les trois phases de l'étude. Le liant hydraulique utilisé pour la technique de stabilisation/solidification est un ciment de type CEM II/B-M (LL-S) 32,5 R (de marque Calcia). Ce ciment est de type Portland composé, répondant aux critères de la norme NF EN 197- | (200 I). || est constitué de 65 à $79 \%$ de clinker, de 21 à $35 \%$ de laitier de haut fourneau et de calcaire, et de 0 à $5 \%$ de constituants secondaires. Sa masse volumique est de l'ordre de $3,03 \mathrm{t} / \mathrm{m}^{3}$ et sa surface spécifique étant de $4000 \mathrm{~m}^{2} / \mathrm{g}$.

Une fois la formulation de traitement définie, les caractéristiques de compactage des mélanges sont déterminées seIon l'essai Proctor normal. Des éprouvettes cylindriques de dimensions $(5 \times 5 \mathrm{~cm})$ sont confectionnées par compression statique à la teneur en eau optimale $\left(w_{\text {OpN }}\right)$ et à $96 \%$ de la masse volumique sèche maximale $\left(\rho_{\mathrm{dOPN}}\right)$. Pour les essais d'aptitude au traitement, 3 éprouvettes sont destinées à la mesure de l'indice du gonflement (Gv) et trois autres à la résistance à la traction indirecte (Rit). Les éprouvettes sont conservées pendant une durée de 4 heures à une humidité relative supérieure à $90 \%$ et à une température de $20 \pm$ $2^{\circ} \mathrm{C}$, avant leur immersion dans un bain thermostatique réglé à $40^{\circ} \mathrm{C}$ pendant une période de cure de 7 jours. Ces conditions de cure accélèrent les réactions d'hydratation au sein du matériau traité et permettent d'entrevoir rapidement une perturbation physico-chimique éventuelle. L'approche méthodologique des essais d'aptitude au traitement est expliquée sur l'organigramme de la Figure 2. L'étude des performances mécaniques à court terme et à long terme a été menée sur des éprouvettes d'élancement $2(\mathrm{D} \times \mathrm{h}=5 \times 10 \mathrm{~cm})$ pour la compression simple (NF P 98-232- I) et d'élancement I (D $\times h$ $=5 \times 5 \mathrm{~cm}$ ) pour la résistance à la traction indirecte (NF P 98232-3). Après la confection, les éprouvettes sont conservées à teneur en eau constante dans des étuis hermétiques. Les essais ont été réalisés à 7, 28, 90, 180 et 360 jours.

\section{Résultats et discussions}

\section{I Point de fixation de la chaux}

La détermination du point de fixation de la chaux (PFC) repose sur la mesure du $\mathrm{pH}$ des sédiments après addition de chaux vive à différents taux par rapport à la masse sèche de sédiment. II s'agit de suivre les variations du $\mathrm{pH}$ des mélanges particules solides-chaux mis en suspension dans l'eau. Les sédiments ont été humidifiés à une teneur en eau de $20 \%$ et conservés pendant 24 heures avant de les traiter à la chaux. Les différents dosages de chaux sont de l'ordre de I à $6 \%$. Un temps de réaction d'une heure est observé avant $\checkmark$ Caractérisation GTR (1992), $\checkmark$ Point de fixation de la chaux.

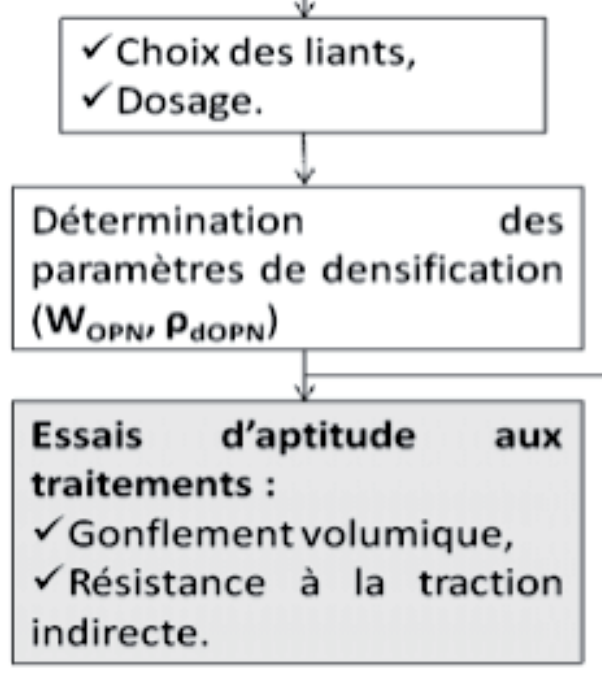

\begin{tabular}{|l|}
\hline Détermination de la \\
résistance \\
à la compression Rc à \\
7,28 et 60 jours. \\
Idem pour 28 jours +32 \\
jours \\
en immersion, 90,180 \\
et 360 jours.
\end{tabular}

Critère de traficabilité : Âge de cure auquel $R_{c} \geq 1 \mathrm{MPa}$

Résistance
l'immersion :
$R_{c}(28+32 j) / R_{c} 60>0,8$
$($ VBS $<0,5)$
$R_{c}(28+32 j) / R_{c} 60>0,6$
$($ VBS $>0,5)$

Détermination de la résistance à la traction indirecte $R_{\text {it }}$ à $7,28,60,90,180$ et 360 jours
Résistance au gel : Âge de cure auquel $R_{\text {it }} \geq 0,25 \mathrm{MPa}$

Figure 2. Démarche pour le traitement des sédiments de dragage pour une valorisation en couche de forme routière (GTS, 2000). 
que les échantillons ne soient humidifiés une deuxième fois, avec un rapport Eau/Solide de 5. Les solutions ainsi obtenues sont mises en agitation pendant I heure avant de mesurer leurs $\mathrm{pH}$ (voir Figure 3). La mesure du $\mathrm{pH}$ a été réalisée selon la norme NF ISO 10390. Les résultats de variation du pH en fonction du dosage de chaux sont montrés sur la Figure 4 Cette figure montre une évolution rapide $\mathrm{du} \mathrm{pH}$ pour des dosages de la chaux entre I et $3 \%$. Au-delà de $3 \%$ le pH se stabilise à une valeur de 12.4 permettant d'obtenir un milieu favorable au développement d'hydrates cimentaires et ainsi au développement de performances mécaniques. C'est cette dernière valeur de la chaux (3\%) qui va être utilisée pour la suite de notre démarche de traitement des sédiments. La Figure 4 montre aussi que la réponse des sédiments au traitement à la chaux est un peu différente. En effet, certains sédiments ( $\mathrm{A0OI}, \mathrm{B} 00 \mathrm{I}, \mathrm{D} 00 \mathrm{l}$ et $\mathrm{G00I}$ ) atteignent la valeur de 12.4 plus rapidement que d'autres sédiments ( $\mathrm{COOI}$, EOO I, FOO I et MOOI). Ceci peut être dû au fait que la matière organique (M.O) présente dans les sédiments consomme une partie de la chaux disponible, ce qui réduit son efficacité pour la neutralisation de l'acidité du milieu.
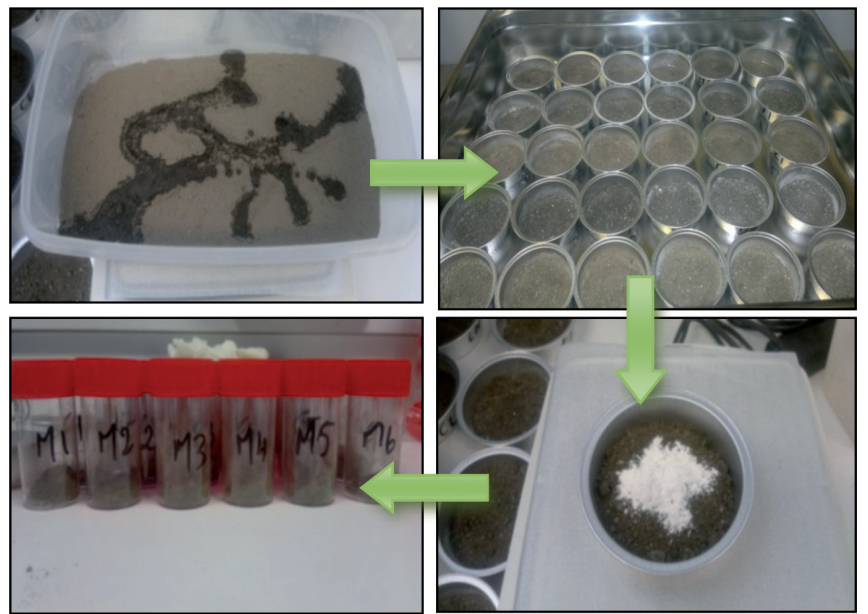

Figure 3. Différentes étapes de préparation des sédiments pour la réalisation de l'essai du point de fixation de la chaux.

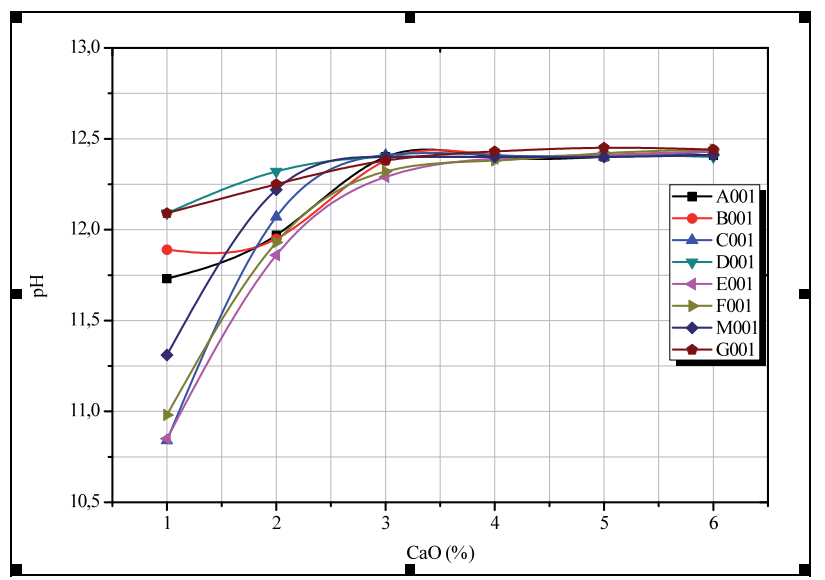

Figure 4. Évolution du pH des huit sédiments étudiés en fonction du pourcentage de chaux.

\subsection{Paramètres de densification}

La deuxième étape avant la réalisation des essais d'aptitude au traitement des sédiments de dragage consiste à déterminer leurs paramètres de densification en adoptant l'essai de compactage Proctor. Les différentes étapes de préparation des sédiments sont montrées à la Figure 5 . Après humidification des sédiments, la chaux est ajoutée après 24 heures. Ensuite, le ciment sera ajouté après une période de I heure pour le temps de réaction de la chaux. Ensuite l'essai de compactage est réalisé après I heure d'ajout du ciment.
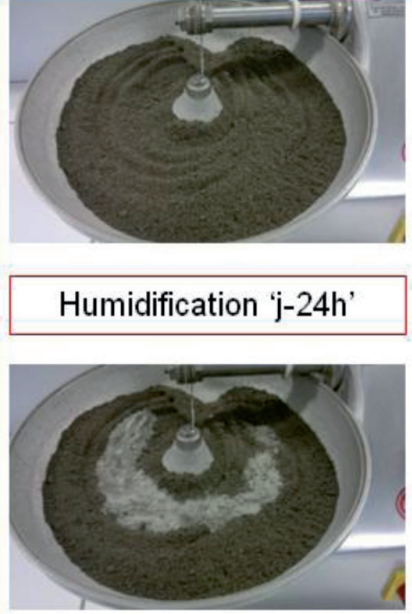

Ajout de 6\% du Ciment CEM II 32,5 'j+1h'

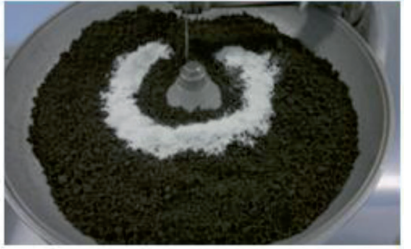

Ajout de $3 \%$ de $\mathrm{CaO}$ 'j'

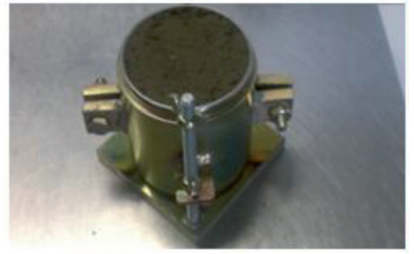

Essai de compactage Proctor Normal 'j+1h'
Figure 5. Différentes étapes de préparation des sédiments traités pour réaliser l'essai Proctor normal.

Les résultats obtenus sont résumés dans le Tableau 2 et les courbes Proctor sont montrées sur la Figure 6. Le Tableau 2 montre qu'on obtient des masses volumiques sèches des sédiments traités comprises entre 1.1 et $1.6 \mathrm{t} / \mathrm{m} 3$ correspondants à des teneurs en eau optimales entre 20 et $40 \%$. Les éprouvettes pour les essais d'aptitude sont confectionnées à partir de ces caractéristiques.

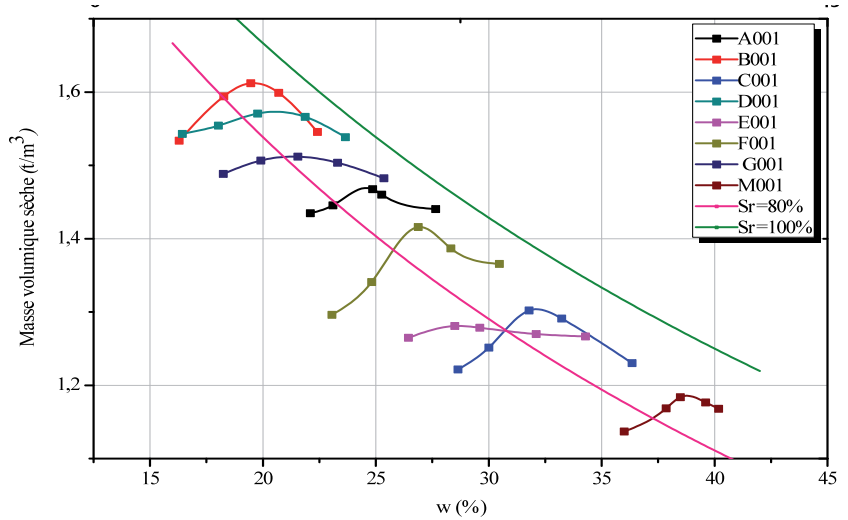

Figure 6. Courbes Proctor Normal des huit sédiments traités. 


\subsection{Confection des éprouvettes}

Selon le GTS (2000), et pour un diamètre des grains maximum (D) inférieur à $2 \mathrm{~mm}$, les éprouvettes ont été moulées par compression statique. Ainsi, deux types d'éprouvettes ont été confectionnées, un premier type (2 éprouvettes de dimensions $5 \times 10 \mathrm{~cm}$ ) pour la mesure de la résistance à la compression simple et un deuxième type d'éprouvettes ( I de dimension $5 \times 5 \mathrm{~cm}$ ) destinées pour mesurer la résistance à la traction indirecte ou diamétrale (voir Figure 7). En ce qui concerne l'essai de gonflement volumique, les éprouvettes sont immergées dans un bain à $40^{\circ} \mathrm{C}$ pendant 7 jours.

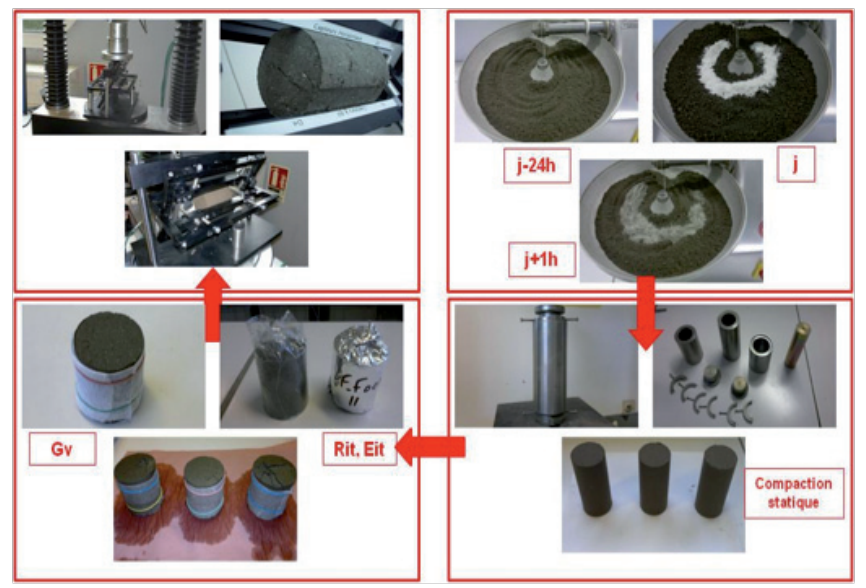

Figure 7. Différentes étapes de préparation des sédiments traités pour réaliser les essais mécaniques.

\subsection{Résultats des essais d'aptitude au traitement}

Le Tableau 3 résume les résultats obtenus pour l'indice de gonflement des huit sédiments traités ainsi que les critères de jugement de leur aptitude au traitement selon le GTS (LCPCSETRA, 2000). D'après le Tableau 3, seuls les sédiments B00 I et $\mathrm{GOO}$ I sont aptes au traitement. En analysant ces résultats, il y a une certaine concordance entre la teneur en matière organique (M.O) et l'indice de gonflement volumique (Gv). Cependant, la présence d'autres éléments perturbateurs (non encore analysés) peut perturber ces essais. C'est le cas des autres sédiments. Pour les sédiments $\mathrm{AOO}$ I et F00 I, il n'était pas possible d'obtenir des mesures car les éprouvettes n'ont pas résisté aux conditions de cure.

Tableau 3. Résultats de gonflements volumiques des huit sédiments traités

\begin{tabular}{|c|c|c|c|}
\hline Sédiments & Gv (\%) & Jugement & M.O (\%) \\
\hline A00I & Non mesurable & Inapte & 10.3 \\
\hline B00I & I & Apte & 4.8 \\
\hline COOI & Non mesurable & Inapte & 14.1 \\
\hline
\end{tabular}

\begin{tabular}{|c|c|c|c|}
\hline Sédiments & Gv (\%) & Jugement & M.O (\%) \\
\hline D00I & 5 & Douteux & 7.6 \\
\hline E0OI & Non mesurable & Inapte & 10.7 \\
F0OI & Non mesurable & Inapte & 11.4 \\
G00I & I & Apte & 5.4 \\
MOOI & Non mesurable & Inapte & 17.1 \\
\hline
\end{tabular}

Les résultats obtenus de la résistance à la traction indirecte après immersion (Rit) sont représentés sur la Figure 8. Un seuil de $0.20 \mathrm{MPa}$ est nécessaire afin de valider l'aptitude d'un sédiment au traitement appliqué. Dans le cas présent, et comme le montre la Figure 8, les huit sédiments ne développent, au cours des 7 jours d'immersion à $40^{\circ} \mathrm{C}$, que des Rit inférieures à $0.1 \mathrm{MPa}$, les définissant ainsi comme étant inaptes au traitement.

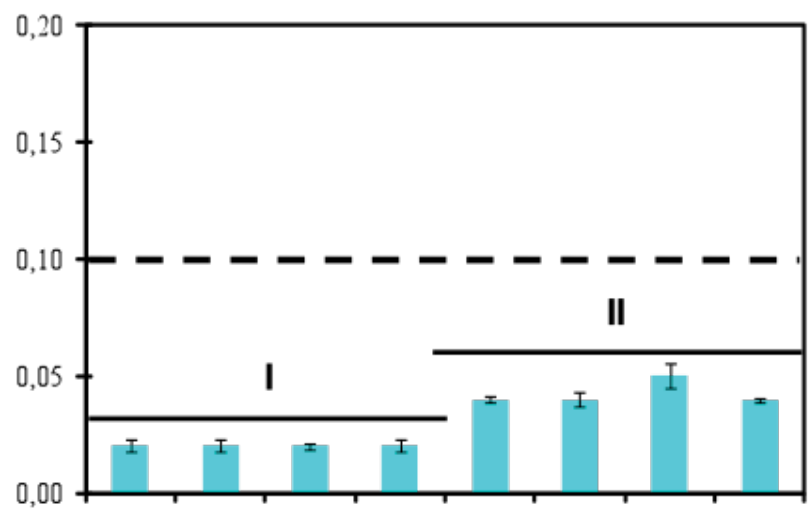

Figure 8. Résistances à la traction indirecte des huit sédiments traités à 7 jours d'immersion.

\subsection{Suivi des performances mécaniques des sédiments traiteés}

Dans la suite, les résultats de suivi des performances mécaniques des sédiments traités sont présentés et discutés. II s'agit de mesurer la résistance à la compression simple et la résistance à la traction indirecte en fonction des temps de cure (7 j, 28 j, 60 j, 90 j, I 180 j et 360 jours). La résistance à la compression simple est un indicateur de l'intégrité physique du matériau solidifié. Dans l'optique d'une valorisation en construction routière, la Rc devrait atteindre I MPa. Par ailleurs, la résistance à la compression diamétrale est un indicateur de la résistance du matériau solidifié à la traction. L'exigence fixée pour une réutilisation du matériau en couche de forme est une Rit de $0.25 \mathrm{MPa}$. Les résultats obtenus sont montrés sur les Figures 9 et I0. La Figure 9 indique que seul le sédiment D00I a atteint le seuil préconisé par le GTS après une période de cure de 28 jours pour la résistance à la compression simple. Une augmentation de la résistance à la compression simple au début des temps de cure est observée. On note une stabilisation des valeurs après 60 jours de cure pour les échantillons $\mathrm{COOI}$, 
E00 I et M00 I. Une légère baisse est observée à 60 jours pour l'échantillon G00I et à 90 jours pour les échantillons B00 I et D00 I. Après 90 jours, la résistance augmente de nouveau pour l'ensemble des sédiments. Ceci peut être dû à l'effet de la réaction pouzzolanique qui permet la formation de nouveaux hydrates à partir des ions de calcium et d'argiles suite au traitement adopté. La présence de M.O et de substances chimiques dans les sédiments peut induire cette différence dans l'évolution de la résistance à la compression simple en fonction du temps.

La Figure 10 montre qu'il y a une augmentation de la résistance à la traction indirecte en fonction du temps puis une tendance vers une stabilisation pour l'ensemble des sédiments traités. Toutefois, les seuils de $0.25 \mathrm{MPa}$ requis pour la résistance au gel n'ont pas été obtenus pour l'ensemble des sédiments traités. Ceci permet de conclure que les sédiments traités à la chaux et au ciment ne peuvent pas être valorisés comme matériau routier. L'une des solutions consiste à ajouter un correcteur granulométrique afin d'améliorer leur propriétés mécaniques. Des essais sont en cours et les résultats feront l'objet d'autres publications.

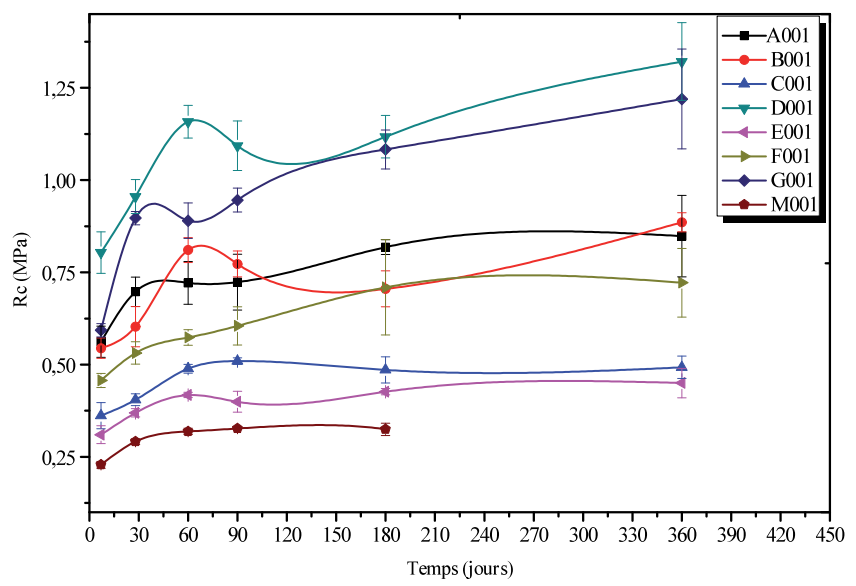

Figure 9. Evolution de la résistance à la compression simple des huit sédiments traités en fonction du temps de cure.

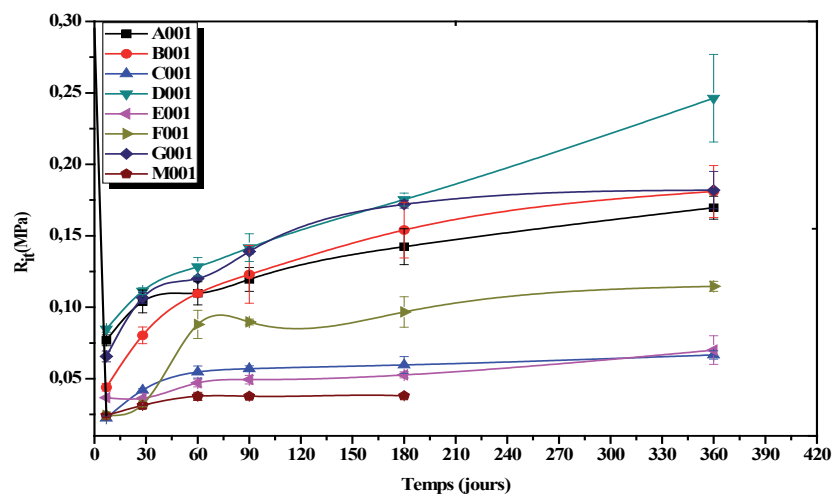

Figure 10 . Evolution de la résistance à la traction indirecte des huit sédiments traités en fonction du temps de cure.

\section{Discussion}

L'observation de l'eau dans laquelle les sédiments ont été conservés pendant une semaine révèle une coloration jaunâtre et la présence de matières en suspensions. Ceci traduit la présence notable de substances humiques au sein des échantillons testés (Cabane et al., 2005). En effet, dans un milieu basique les acides humiques et fulviques réagissent et deviennent solubles (Rekik, 2007). Cela peut être la cause de la déstructuration et les faibles résistances des éprouvettes de sédiments pour lesquelles les teneurs en M.O. dépassent les 10\%. Le gonflement volumique des sédiments traités peut être causé aussi par la présence d'éléments chimiques tels que les sulfates (Sulfates de Calcium) et les sulfures (Pyrite). En effet, la présence de sulfates ( $\mathrm{CaSO} 4$ ) permet la formation de nouveaux produits tels que l'ettringite qui provoque des gonflements importants. D'autre part, la présence de sulfures (FeS2) conditionne la formation des sulfates. En effet, Cabane et al. (2005) explique que les quantités des sulfates dans le milieu dépendent aussi de la vitesse d'oxydation du sulfure. Cette oxydation est fonction des conditions de conservation des échantillons. Les sédiments COOI, E0OI, F0OI et M0OI présentent les taux les plus élevés de sulfures et sulfates et ce sont les sédiments qui n'ont pas résisté aux conditions de conservation de l'essai de gonflement. Par ailleurs, le sédiment G00I avec des taux considérables de sulfates et de sulfures donne des résultats satisfaisants par rapport au critère de gonflement. Cela peut être expliqué par ces caractéristiques physiques (granulométrie, surface spécifique, porosité, compacité) qui défavorisent l'oxydation des sulfures.

\section{CONCLUSION}

II est fait état des premiers résultats de traitement engagé pour une valorisation des sédiments de dragage en couche de forme, reposant sur le GTR et le GTS. Après une classification géotechnique des sédiments les regroupant dans les classes A I et $\mathrm{A} 2$ de sols fins, une formulation de traitement composée de $3 \%$ de chaux vive et $6 \%$ de CEMII 32,5 a été appliquée. Le premier essai prescrit par le GTS avant de poursuivre une étude plus complète en laboratoire pour un matériau traité aux liants hydrauliques est l'essai d'aptitude au traitement. Les résultats obtenus pour huit sédiments révèlent une inaptitude des sédiments bruts à leur réutilisation en techniques routières pour cette formulation recommandée par le GTS. Ceci suggère que pour répondre aux exigences du GTS en terme de performances mécaniques à court et à long terme, il faudra tenir compte des propriétés des sédiments et rechercher un traitement adapté. C'est à quoi le projet SETARMS devra répondre pour envisager de façon pratique une valorisation des sédiments de dragage. Une des solutions serait d'ajouter un correcteur granulaire afin d'améliorer la compacité des sédiments et améliorer leur réponse au mode de traitement choisis. Ceci fera l'objet des prochaines études afin d'envisager de façon pratique une valorisation des sédiments de dragage. 


\section{Références bibliographiques}

Projet SETARMS : www.setarms.org.

CABANE N., 2008, Sols traités à la chaux et aux liants hydrauliques: Contribution à l'identification et à l'analyse des éléments perturbateurs de la stabilisation, Thèse de doctorat, École des mines d'Alès, France.

COLIN D., 2003, Valorisation des sédiments fins de dragage en technique routière,Thèse de Doctorat, Université de Caen, France.

DUBOIS V., 2006, Etude du comportement physico-mécanique et caractérisation environnementale des sédiments marins, Thèse de Doctorat, Université d'Artois, France.

EADS J.L., GRIM P.E., 1966. A quick test to determine lime requirements for lime stabilization. Highway Res. Rec., n 139, pp : 61- 72.

LEVACHER D., COLIN D., PERRONI A.C., DUAN Z. et SUN L. 2006, recyclage et valorisation de sédiments fins de dragage à usage de matériaux routiers. IXème Journées Nationales Génie Civil-Génie Côtier, Brest, France, pp : 603-612

LCPC-SETRA, 1992, Réalisation des remblais et des couches de forme (GTR) - Guide technique. Fascicules I et II, 200 p.

LCPC-SETRA, 2000, Traitement des sols à la chaux et/ou aux liants hydrauliques (GTS) - Application à la réalisation des remblais et des couches de forme - Guide technique, $240 \mathrm{p}$.

LEVACHER D., SANCHEZ M., DUAN Z., LIANG Y. 20 I I.Valorisation en unité pilote de sédiments méditerranéens : étude des caractéristiques géotechniques et de la perméabilité. Revue Paralia, Vol. 4, pp : 4.I - 4.20.

MAHERZI W., 20I3, Valorisation des sédiments de dragage marins traités aux liants hydrauliques en techniques routières, Thèse de Doctorat, Université de Caen, France.

REKIK B., 2007, Propriétés géotechniques des sédiments de dragage traités au ciment, Thèse de doctorat, Université de Caen, France.

SANNIER L., LEVACHER D. et JOURDAN M., 2009. Approche économique et validation de méthodes de traitements aux liants hydrauliques de sédiments marins contaminés. Revue Paralia, $n^{\circ} 2, p p: s 2.1-s 2.15$.

ZRI A., ABRIAK N.E. et ZENTAR R., 20 I I. Caractérisation physico-mécanique d'un sédiment brut et traité à la chaux. EJECE, n² 2, pp : 239-267. 